\title{
TNP-470 Inhibits 7,12-Dimethylbenz[a]anthracene- Induced Mammary Tumor Formation When Administered Before the Formation of Carcinoma In Situ but Is Not Additive with Tamoxifen
}

\author{
Sue C. Heffelfinger, Robin B. Gear, Joanne Schneider, Kathy LaDow, Mei Yan, \\ Fengxian Lu, Amy L. Pyle, and David Warshawsky \\ Departments of Pathology (SCH, RBG, JS, MY, FL, ALP) and Environmental Health (KL, DW), University of \\ Cincinnati, Cincinnati, Ohio
}

\begin{abstract}
SUMMARY: In many women pathologic lesions, such as hyperplasia and carcinoma in situ, precede invasive breast cancer. We have shown that tissue vascularity increases with histologic progression to invasive disease. Similarly, in the well-characterized 7,12-dimethylbenz[a]anthracene (DMBA) model of mammary tumorigenesis, preinvasive lesions exhibit increased vascularity with progression. Using this model we asked whether inhibition of angiogenesis would block progression and if so, at which stage. We treated rats with DMBA followed by the potent angiogenic inhibitor, TNP-470, and/or tamoxifen starting 1 day or 6 weeks later. Histopathology and in vitro angiogenic potential of mammary organoids were evaluated 3 months after DMBA. All statistical tests were two-sided. Early TNP-470 and tamoxifen treatment inhibited the formation of carcinoma in situ $(p<0.001)$ and invasive disease $(p<0.001)$. However, their effects were not additive, despite their unique mechanisms of action. TNP-470 administration begun at the time of microscopic carcinoma in situ formation was unable to prevent the further development of carcinoma in situ or invasive breast cancer, whereas tamoxifen was highly effective $(p=0.001)$. There was no added benefit of combining TNP-470 and tamoxifen. TNP-470 therapy, unlike tamoxifen, did not inhibit the angiogenic potential of DMBA-treated normal mammary organoids, supporting its lack of a direct effect on the epithelium. These data provide proof-in-principle that inhibition of angiogenesis early in mammary tumorigenesis prevents mammary tumor formation in a hormone-sensitive model, indicating that angiogenesis is a potential target for cancer chemoprevention. Interactions with other chemopreventive strategies and the timing of administration must be thoroughly examined in vivo. (Lab Invest 2003, 83:1001-1011).
\end{abstract}

\begin{abstract}
A ngiogenesis is the process of forming new vessels from preexisting vessels and is required for growth and metastasis of invasive tumors (Folkman, 1990). Preinvasive pathologies, such as hyperplasia and carcinoma in situ (CIS), that precede invasive tumors are the result of cumulative genetic and epigenetic events. In vitro studies have shown that angiogenesis is a phenotype common to oncogenic mutations (Kerbel et al, 1998). For most tumor types, angiogenesis precedes the invasive phase of the disease, suggesting that the ability to stimulate new vessel growth is an early event in oncogenesis (Folkman et al, 1989; Kohn and Liotta, 1995). If precursor pathologies are dependent upon angiogenesis for progression, then inhibition of angiogenesis becomes an attractive target for chemoprevention. However, the stages of tumor formation that are dependent upon angiogenesis are unknown.
\end{abstract}

DOI: 10.1097/01.LAB.0000075641.27128.67

Received January 13, 2003.

This study was funded by the National Cancer Institute (R01-CA82996). Address reprint requests to: Dr. S. C. Heffelfinger, PO Box 670529, Department of Pathology, University of Cincinnati, Cincinnati, Ohio 45367-0529.E-mail: sue.heffelfinger@uc.edu
The purpose of this study was to determine which stages of breast tumor formation are dependent upon angiogenesis for development and to compare the chemopreventive effects of an angiogenic inhibitor to those of tamoxifen. In women and rodent model systems, angiogenesis occurs before the development of histologic changes of the breast (Heffelfinger et al, 1996, 2000; Lichtenbeld et al, 1998), suggesting that oncogenic events indicative of risk may be predicted by an angiogenic phenotype. Furthermore, even the earliest precursor lesions may be dependent upon angiogenesis for development. To study this question, we used the well-characterized 7,12dimethylbenz[a]anthracene (DMBA) rat mammary system (Huggins et al, 1961) in which a single oral dose of DMBA induces histologic changes within a 3-month time frame that recapitulate the series of morphologic precursors seen in women with breast cancer. As in women, tumor production is dependent upon ovarian hormones (Hollingsworth et al, 1998), and all precursor stages are angiogenic, with the maximal vascular density found associated with intraductal proliferations (IDP) (Heffelfinger et al, 2000). Therefore, a significant up-regulation in angiogenesis occurs before the expansion in epithelial volume seen with the 
formation of palpable CIS. Using this model, we asked whether inhibition of angiogenesis would hinder mammary tumor formation when administered early in the process of tumorigenesis or when administered at the time of maximal epithelial expansion, ie, the conversion of IDP/microscopic CIS to palpable CIS. These data were compared with tumor inhibition by tamoxifen, the most commonly used breast chemopreventive agent in women and a known inhibitor of tumor formation in this model system (Jordan and Allen, 1980). Our data show that inhibition of angiogenesis prevents CIS and invasive breast cancer (INV) formation, when started early, but not late, in progression with an efficacy similar to tamoxifen. However, the effect of a combination of an angiogenic inhibitor and tamoxifen is not additive. These data provide proofof-principle that angiogenic inhibitors can prevent breast tumor formation.

\section{Results}

\section{In Vivo Dose Optimization}

The optimal dose and schedule of TNP-470 and tamoxifen delivery are inconsistent in the literature. We tested various doses and schedules in rats, age and weight matched to those that would be used in future studies, by performing an in vivo Matrigel pellet assay. After 14 days of drug treatment, the area of vascular influx into each pellet was determined and plotted as mean ( \pm SD) percent vascularity, setting the vascularity of vehicle control-treated rats at $100 \%$ (n $=5$ ) (Fig. 1). The asterisk indicates a difference relative

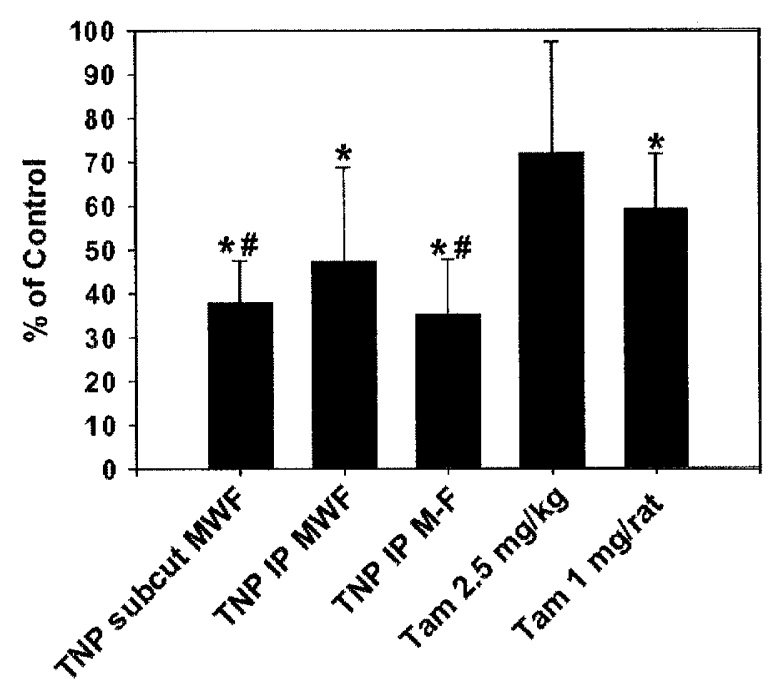

Figure 1.

Optimization of TNP-470 and tamoxifen dosing schedule using the Matrigel pellet assay. TNP-470 (TNP) was administered at $30 \mathrm{mg} / \mathrm{kg}$ three times per week sc (TNP subcut MWF) or intraperitoneally (TNP IP MWF). In the third group, TNP-470 was administered at $15 \mathrm{mg} / \mathrm{kg}$ five times per week intraperitoneally (TNP IP M-F). Tamoxifen was administered once weekly as a sc injection in corn oil at either $2.5 \mathrm{mg} / \mathrm{kg}$ or $1 \mathrm{mg} / \mathrm{rat}$ (approximately $5.5 \mathrm{mg} / \mathrm{kg}$ ). Data are plotted as mean ( $\pm \mathrm{SD}$ ) percent vascularity, setting the vascularity of the vehicle control rats at $100 \%$ ( $n=5$ rats per group). ${ }^{*}$ Significant difference relative to vehicle controls $(p<0.05)$; \#significant difference relative to the 2.5 $\mathrm{mg} / \mathrm{kg}$ dose of tamoxifen $(p<0.05)$. to vehicle control-treated rats $(p<0.05)$. Consistent with reports in the literature, TNP-470 delivered at 30 $\mathrm{mg} / \mathrm{kg} 3$ times per week inhibited vessel growth into the pellet, either when delivered sc or ip (Kruger and Figg, 2000). Dividing the dose during 5 days did not significantly improve efficacy. For our inhibitor studies, we were interested in comparing an angiogenic inhibitor that functions primarily on the endothelium (TNP470) with an agent that primarily affects the epithelium (tamoxifen). However, in vitro tamoxifen also inhibits endothelial growth (Gagliardi et al, 1996). As shown in Figure 1, $1 \mathrm{mg}$ of tamoxifen per rat (approximately 5.5 $\mathrm{mg} / \mathrm{kg}$ ) injected weekly significantly inhibited vessel ingrowth into the pellet $(p<0.05)$. However, 2.5 $\mathrm{mg} / \mathrm{kg}$ tamoxifen had no effect on vessel growth and was, therefore, chosen for further studies.

\section{TNP-470 and Tamoxifen Inhibit Invasive and Precursor Pathologies Without Synergism}

Although there are some data showing that angiogenic inhibitors block tumor formation when given at the time of early tumor formation in this model system (Perletti et al, 2001), there are no data examining the effect of angiogenic inhibition on precursor pathologies nor are there data examining the combined effects of an angiogenic inhibitor with an estrogen receptor antagonist. To test this we performed the following experiment. Within the first week after DMBA administration and continuously thereafter, rats were treated with TNP-470 (30 mg/kg sc 3 times weekly), tamoxifen $(2.5 \mathrm{mg} / \mathrm{kg}$ weekly), or both. At these dosages TNP-470 inhibits vascular growth relative to tamoxifen in the Matrigel pellet assay $(p<0.05$, Fig. 1). Histologic analyses were performed at 3 months after DMBA, at which time $100 \%$ of the DMBA treatment-alone group had advanced CIS, INV, or both. Criteria for histologic diagnosis were according to Russo and Russo (2000). Histologic sections of typical DMBA-induced pathologies are shown in Figure 2. Data for analysis were pooled from three separate studies. Tumor frequencies were essentially the same for each study. Group sizes in the pooled studies are as follows: DMBA (32), TNP-470 (27), tamoxifen (11), and TNP-470 + tamoxifen (11). When administered before the formation of histopathologic lesions, both TNP-470 and tamoxifen reduced the number of CIS (73\% and $79 \%$, respectively) and INV ( $81 \%$ and $96 \%$, respectively) per rat $(p<0.001)$, with the combination of therapies yielding results similar to tamoxifen alone ( $p<0.001)$ (Fig. 3A). The decreases in tumor number correlated with a decreased total tumor burden with all treatments, which was statistically significant for invasive disease $(p<0.05)$ (Fig. 3B). There were no significant differences in individual tumor sizes among the groups (Fig. 3C), although the number of tumors in the tamoxifen treatment group is too small for statistical analysis. Both TNP-470 and tamoxifen decreased the proportion of these groups with INV $(p<0.05)$ (Fig. 3D). TNP in all experiments delayed the time of first tumor formation by approximately 10 days, a result that did not reach statistical 


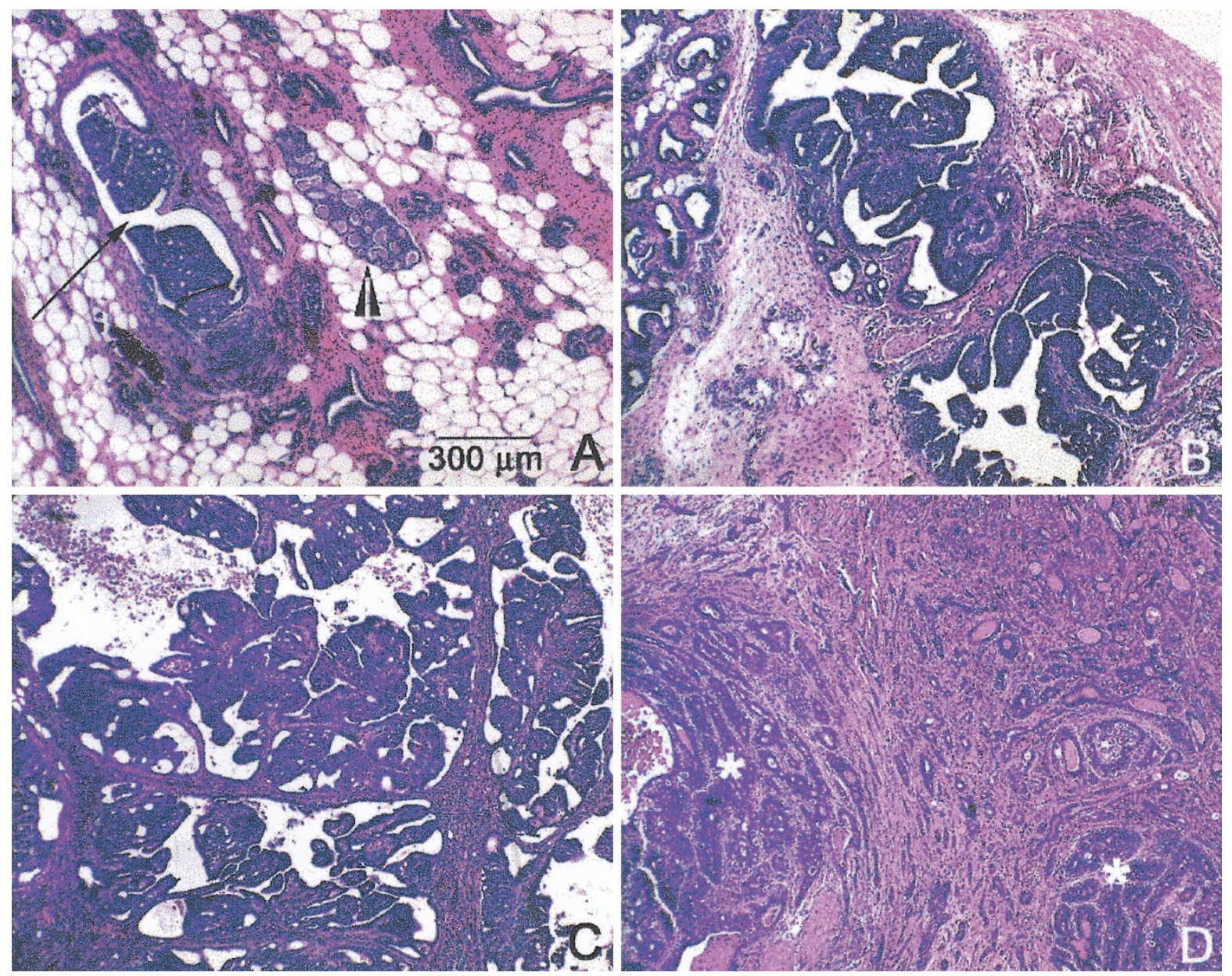

Figure 2.

7,12-Dimethylbenz[a]anthracene (DMBA) induces proliferative lesions that are both ductal (IDP) and lobular (lobular HP) in morphology. A, a typical IDP (arrow) and a hyperplastic lobule (arrowhead). B, a small cluster of IDP. In this model system, carcinoma in situ (CIS) has a number of different morphologies. C, a typical CIS with a papillary morphology. Unlike human invasive breast carcinoma, invasive breast cancer (INV) in this model system is never seen absent a CIS component to the tumor. D, highly invasive tumor cells surrounding residual $\mathrm{CIS}\left({ }^{*}\right)$ and expanding into the adjacent stroma. All histologic sections were stained with hematoxylin and eosin and acquired using a $\times 4$ objective.

significance (data not shown). However, tamoxifen delayed time to first tumor by 20 days, which was significantly delayed beyond tumor formation in the DMBA group $(p<0.05)$. In all cohorts $100 \%$ of animals had one or more types of pathology, except the tamoxifen group in which 2 of 11 rats had no pathologic mammary lesions (not shown). In summary, TNP-470 inhibited the number of CIS and INV per rat and the mean total tumor burden and percentage of animals with INV, with numbers that were comparable to those of tamoxifen. Note that both TNP-470 and tamoxifen inhibited the formation of lobular hyperplasia but not the hyperplastic ductal lesions that are precursors to invasive disease (IDP) (Fig. 3A).

\section{Maximal Tissue Proliferation and Vascularity Are Not Different with TNP-470 or Tamoxifen Treatment, but TNP-470 Inhibited the Epithelial Rate of Apoptosis}

Despite development in the presence of angiogenic inhibition, the few tumors formed in the long-term treatment group were able to grow to a size comparable to untreated DMBA-alone controls. To study mechanisms by which this might occur, we examined maximal proliferative rates in the vasculature and the epithelium and the apoptotic rate within the epithelium. Neither TNP-470 nor tamoxifen altered the maximal proliferative rate of CIS or INV, the maximal microvascular density, bromodeoxyuridine (BrdU) vascular area, or total vascular area (not shown). Therefore, although total tumor number and volume per rat were decreased in the treated groups, those tumors that formed were equivalent to the DMBAalone cohort in size and maximal vascularity. This is best illustrated by a stained histologic section of a tumor from a DMBA-treated (Fig. 4A) or DMBA plus TNP-470-treated (Fig. 4B) rat. Note that numerous small vessels (brown) stream among islands of epithelium. In both cases there is considerable epithelial BrdU incorporation (blue) and numerous proliferating vessels (arrow). However, the apoptotic rate in the epithelium was significantly decreased in both CIS ( $p$ 

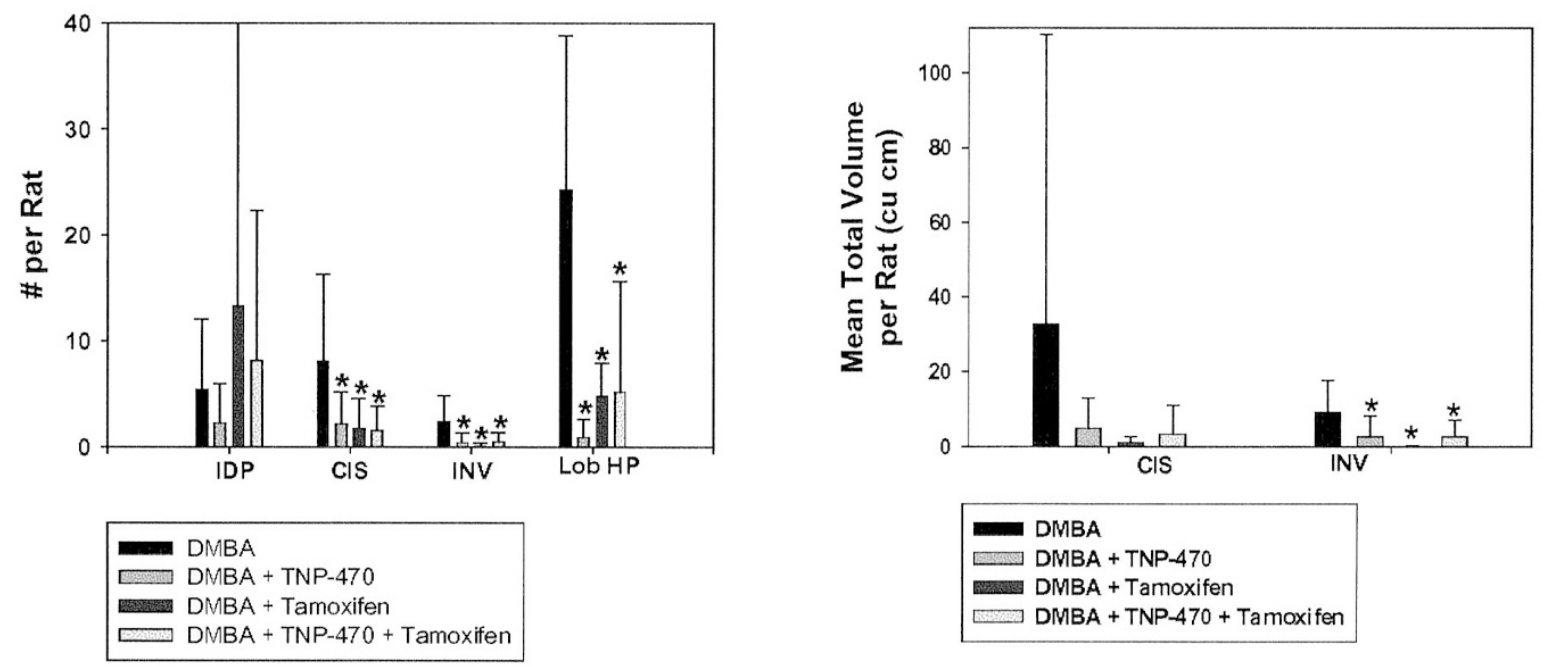

A
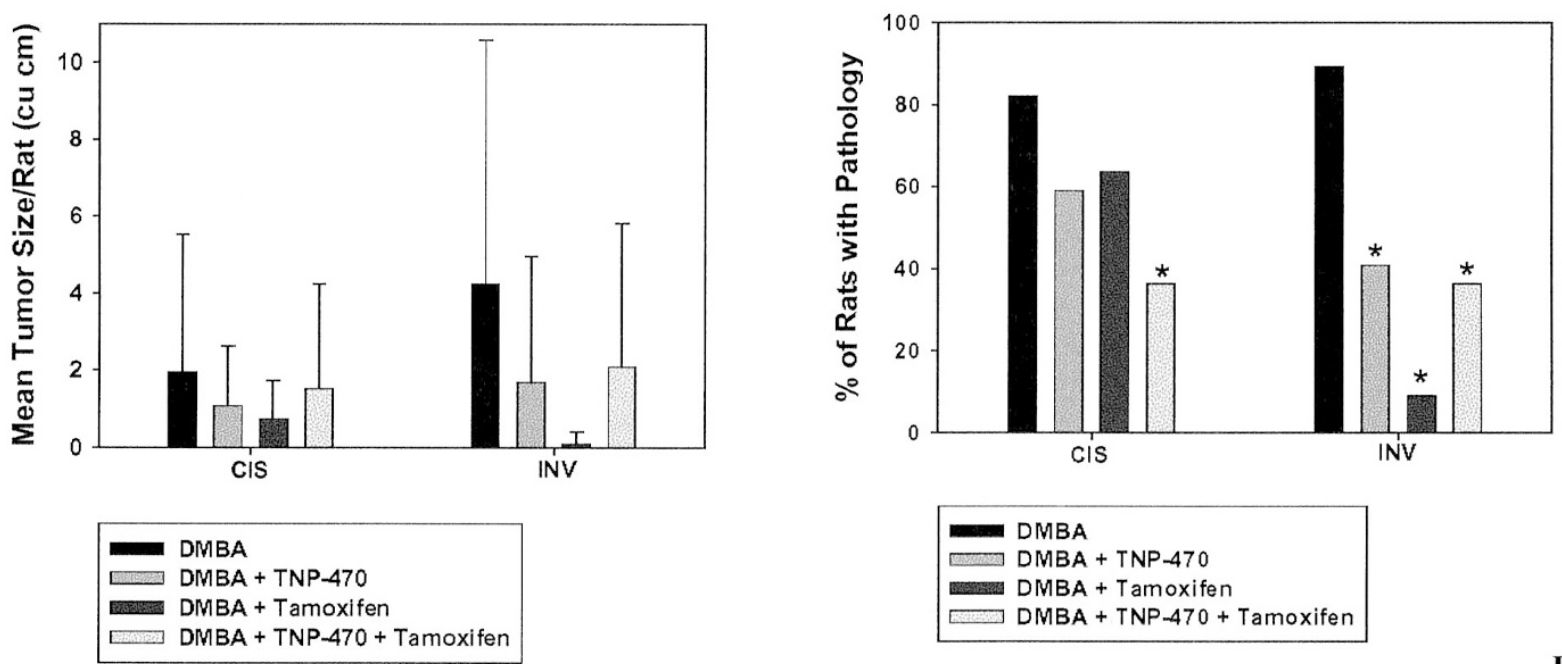

$\mathrm{C}$

Figure 3.

TNP-470 and tamoxifen inhibition of mammary precursor lesions and tumors with early intervention. Pathologies were characterized as IDP, CIS, INV, or lobular HP. Data represent mean $( \pm \mathrm{sD})$ from each treatment group: DMBA alone (DMBA), DMBA and $30 \mathrm{mg} / \mathrm{kg}$ TNP-470 (DMBA + TNP-470), DMBA and $2.5 \mathrm{mg} / \mathrm{kg}$ tamoxifen (DMBA + Tamoxifen), DMBA and $30 \mathrm{mg} / \mathrm{kg}$ TNP-470 and $2.5 \mathrm{mg} / \mathrm{kg}$ tamoxifen (DMBA + TNP-470 + Tamoxifen). TNP-470 and tamoxifen were delivered beginning within 1 week of DMBA administration. * Significantly $(p<0.05)$ different from DMBA-alone treatment. Plotted are number of each pathology per rat $(A)$, total tumor volume per rat $(B)$, mean tumor size per rat $(C)$, and the percentage of rats bearing each type of pathology (D).

$<0.05)$ and INV $(p<0.05)$ in the TNP-470 treatment group relative to the DMBA-alone group (Fig. 5).

\section{TNP-470 Administration at the Time of Early CIS Formation Does Not Decrease the Incidence of CIS or INV}

To determine when IDP and microscopic CIS were formed after DMBA exposure, we followed sets of animals over time to characterize the mammary histopathology. In a number of studies, IDP and occasionally even microscopic and small palpable CIS are present by 6 weeks after DMBA. Therefore, this time point was chosen to represent individuals with proliferative ductal pathologies and CIS. Figure 6 shows data from one study in which we compared patholo- gies at 6 weeks and 12 weeks after DMBA. Note that the number of IDP remains constant over time, whereas the number of CIS increases, as does the number of lobular hyperplasias $(p<0.05)$. INV is found in only the 12-week group.

To further define at which stages TNP-470 may be acting, we administered TNP-470, tamoxifen, or both (12 rats per group) 6 weeks after DMBA exposure. When administered at this transition of proliferative lesions or microscopic CIS to palpable CIS, TNP-470 had no effect on the frequency of CIS or INV. Tamoxifen inhibited CIS relative to either the DMBA or TNP-470 cohort ( $p=0.001$ ) (Fig. 7A). Tamoxifen also inhibited INV relative to the TNP-470 cohort $(p=$ 0.001) (Fig. 7A). Although the combination of tamox- 


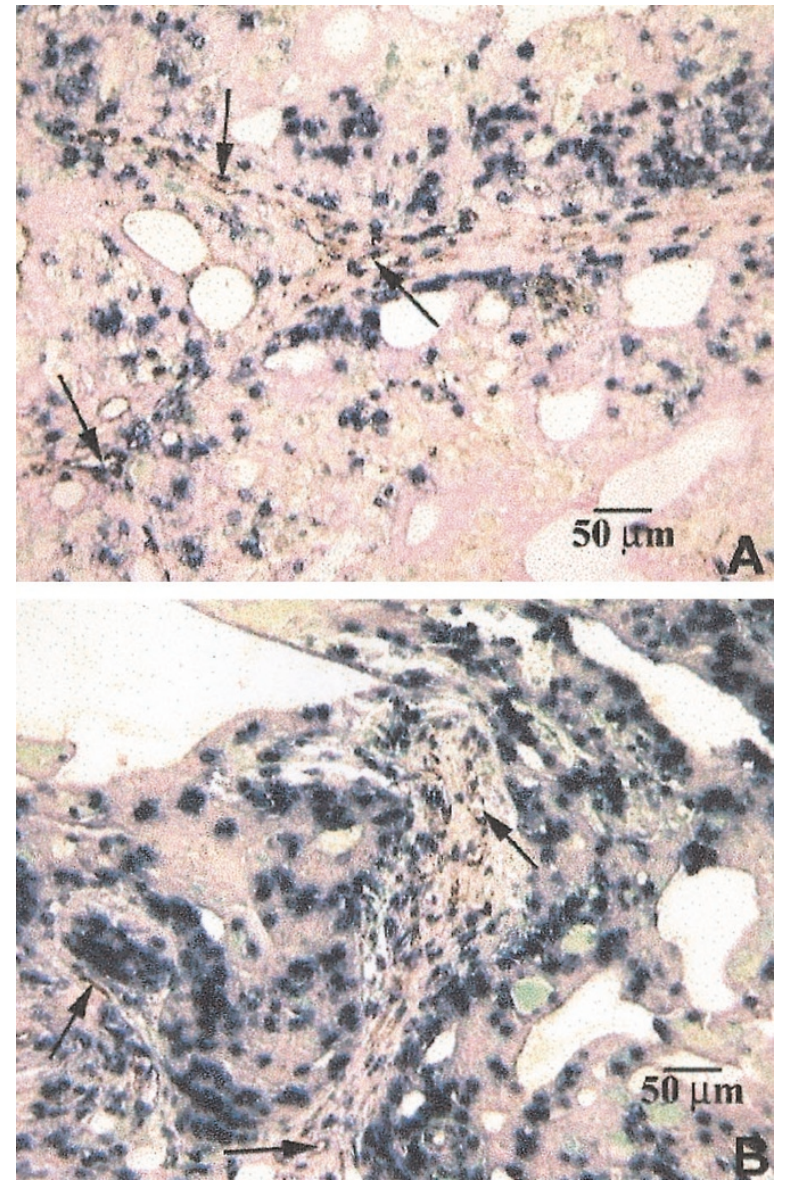

Figure 4.

Histologic section of CIS from a DMBA-treated rat (A) and from a DMBAtreated rat that also received 3 months of TNP-470 after initiation (B). Images shown here are taken with $\mathrm{a} \times 20$ objective. Each tumor was stained using an antibody to bromodeoxyuridine (BrdU) (blue) and von Willebrand factor (VWF) (brown). Some of the vessels that also show nuclear staining for BrdU in the endothelium are indicated with an arrow.

ifen and TNP-470 had no statistically significant effect on CIS, having a value intermediate to that of either treatment alone, the combination did inhibit INV relative to the TNP-470 group $(p=0.001)$. Tamoxifen and the combination of TNP-470 and tamoxifen reduced CIS tumor volume per rat relative to the TNP-470 cohort $(p<0.05)$ but had no effect on the volume per rat of INV (Fig. 7B). This is reflected in the large size of some INV in the tamoxifen group (Fig. 7C). Note that the lack of statistical significance for tumor size in the combined treatment group is a result of the small number of INV present. The percentage of animals bearing CIS or INV were unaffected by these treatments (Fig. 7D). We addressed the concern regarding whether TNP-470 was functioning in these animals by measuring the frequency of lobular hyperplasia, a DMBA-induced pathology unrelated to adenocarcinoma formation that ultimately progresses to tubular adenomas (Russo et al, 1989). All treatment groups inhibited the formation of lobular hyperplasia $(p<$ 0.001) (Fig. 7A). In summary, whether measuring tumor number, total volume, or tumor size, TNP-470 administered at the transition of proliferative lesions or microscopic CIS to palpable CIS had no tumor preventive effect for ductal lesions relative to the untreated DMBA cohort.

\section{In Vivo Tamoxifen, but Not TNP-470, Inhibits the Angiogenic Potential of Normal Organoids}

We have previously shown that normal mammary organoids from DMBA-treated rats are angiogenic in vitro (Heffelfinger et al, 2000). If the mechanism of TNP-470 and tamoxifen functions primarily on the endothelium and epithelium, respectively, we predicted that histologically normal mammary organoids from TNP-470-treated rats would have the same angiogenic capacity in vitro as the DMBA cohort, but normal organoids from tamoxifen-treated rats would have a reduced angiogenic capacity in vitro because of tamoxifen's direct effects on the epithelium. Therefore, we examined the angiogenic potential of normal mammary organoids 3 months after carcinogen exposure from DMBA-treated rats and compared them with mammary organoids from rats that received DMBA plus TNP-470, tamoxifen, or both for 3 months (Fig. 8A). Angiogenic potential was measured as the induction of endothelial tubules in collagen gels. Skeletal muscle from DMBA-treated rats was assayed as a negative control. Data are plotted as relative tubule length, as a percent of tubule length induced by purified human vascular endothelial cell growth factor (VEGF). Presented are means from individual organoid isolates from four rats in each group, five wells per rat, analyzed in two separate experiments. Histologically normal mammary organoids from DMBA + TNP-470 treated rats were equally angiogenic compared with DMBA alone ${ }^{*} p<0.001$ relative to control rats or skeletal muscle from DMBA-treated rats), whereas organoids from tamoxifen-treated rats were less angiogenic. The addition of in vivo TNP-470 to tamoxifen had no effect relative to tamoxifen on the in vitro angiogenic capacity. Histologic examination of organoid preparations confirmed that the tissues were primarily epithelial with occasional scant amounts of intralobular stroma (Fig. 8B). BrdU labeling of organoids in vitro between the two groups was also the same (Fig. 8B).

\section{Discussion}

We chose TNP-470 for these studies because of its efficacy in the therapy of other hormone-dependent rat tumors and because it is in clinical trials as a therapeutic agent for breast cancer (Takechi et al, 1994). Because any antiangiogenic agent must be compared with current treatments, we compared the efficacy of TNP-470 and that of the commonly prescribed breast cancer chemopreventive agent, tamoxifen. Unlike TNP-470, which functions primarily on the endothelium (Ingber et al, 1990), tamoxifen has complex effects on angiogenesis, acutely increasing VEGF, while directly inhibiting vessel growth (Gagliardi et al, 1996; Ruohola et al, 1999). To discriminate among these activities, we obviated the direct effect of 
CIS

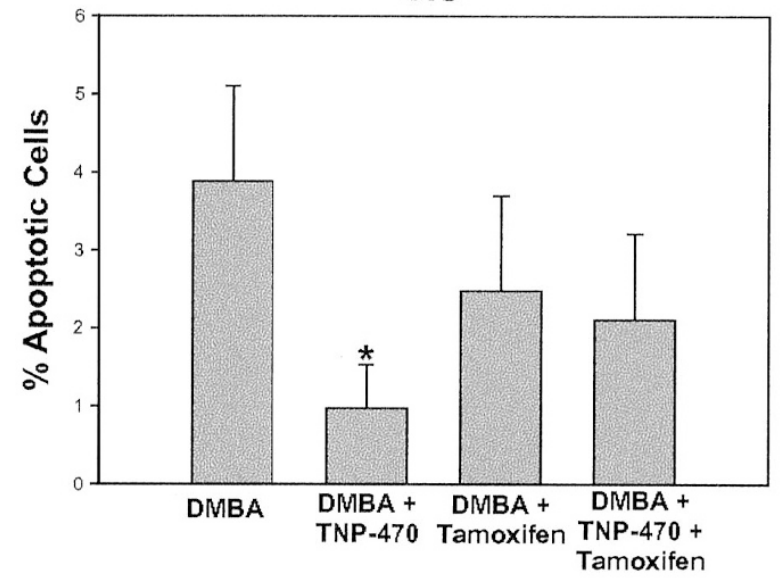

INV

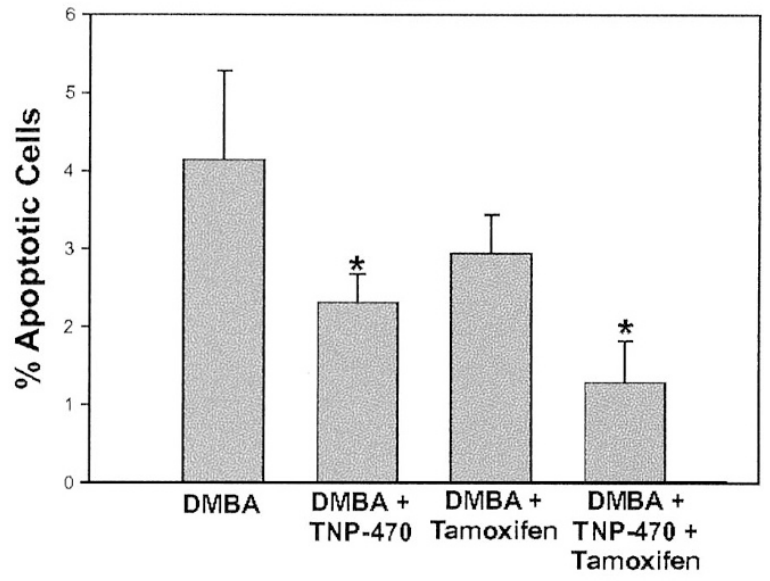

A

B

Figure 5 .

Epithelial apoptotic rate in CIS and INV from each treatment group as determined by terminal deoxynucleotidyl transferase-mediated dUTP nick-end labeling Treatment groups are as described in Figure 2. TNP-470 decreased the apoptotic rate relative to the untreated DMBA group in CIS $(\mathrm{A})\left({ }^{\star} p=0.005\right)$ and INV (B) $\left({ }^{\star} p\right.$ $<0.05)$.

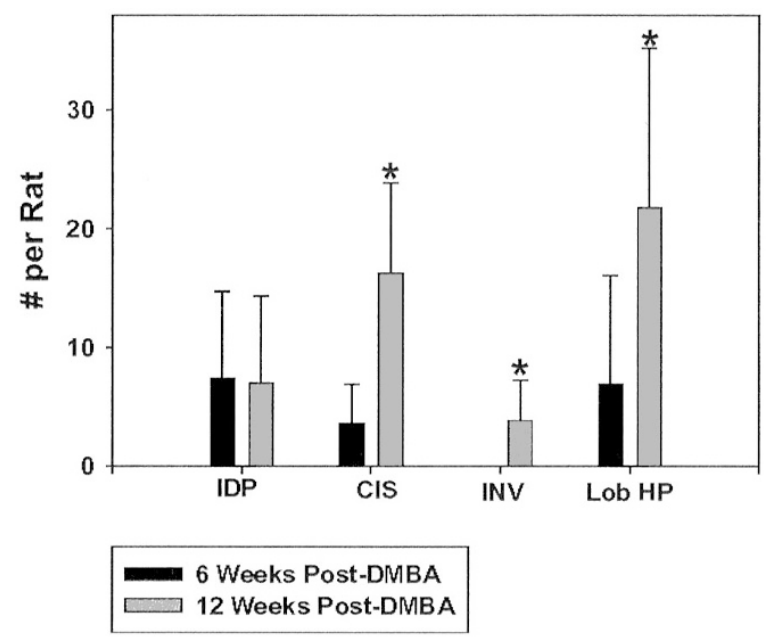

Figure 6 .

Histologic analyses were performed on mammary glands of DMBA-treated rats 6 weeks and 12 weeks after initiation. Shown are the mean ( \pm SD) for each type of pathology. ${ }^{*}$ Significant difference by $t$ test between the two groups at each time point $(p<0.05)$.

tamoxifen on the vasculature by choosing a tamoxifen concentration that did not inhibit growth factor-induced angiogenesis in vivo.

\section{The Stage of TNP-470 Action Precedes the Formation of $\mathrm{ClS}$}

In this study we treated rats with an angiogenic inhibitor after DMBA initiation and examined CIS and INV formation. Inhibition of angiogenesis by TNP-470 administration early in the process of tumorigenesis resulted in a significant decrease in the number of CIS and INV tumors and total tumor burden per rat. The question remains as to how TNP-470 affects the decrease in the number of CIS and INV. Given that CIS and INV were inhibited by roughly the same percentage, one could argue that TNP-470 had no effect on the progression of CIS to INV and that the decrease in INV was entirely a result of a stochastic decrease in the precursor CIS. (Note that unlike human invasive breast tumors, INV in the DMBA model is found only as an outgrowth of CIS, ie, after examining tumors from a total of 510 DMBA-treated rats, to date INV has always been found intimately associated with a large component of CIS within the same tumor.) TNP-470 inhibited the IDP precursor by $59.9 \%(p=0.028)$, a value that lost statistical significance when the tamoxifen data were added to the pool. Given that IDP has the highest relative vascularity (vessel area/epithelial area) of all mammary pathologies in this model (Heffelfinger et al, 2000), we hypothesized that TNP-470 acts at the stage of IDP formation. Although this hypothesis is not fully supported by the first set of data, the experiment in which TNP-470 was administered after the formation of IDP does support this hypothesis. If TNP-470 has its major effect during the epithelial expansion of IDP to CIS, then the administration of TNP-470 at 6 weeks after DMBA would have resulted in diminished CIS formation. This was not the case. Therefore, we suggest that TNP-470 has a narrow window of activity in the early angiogenic phase of tumorigenesis.

The supposition that not all angiogenic beds are equally susceptible to specific angiogenic inhibitors is supported by the work of Perletti et al (2001) in which endostatin was given for 28 days to DMBA-treated rats at the time of initial tumor development. Shortterm endostatin treatment significantly inhibited the number and total volume of tumors per rat, unlike TNP-470 in our study. However, after the cessation of endostatin therapy, new tumors formed but only at sites different from those in which previous tumors had been found (Perletti et al, 2001), suggesting that 

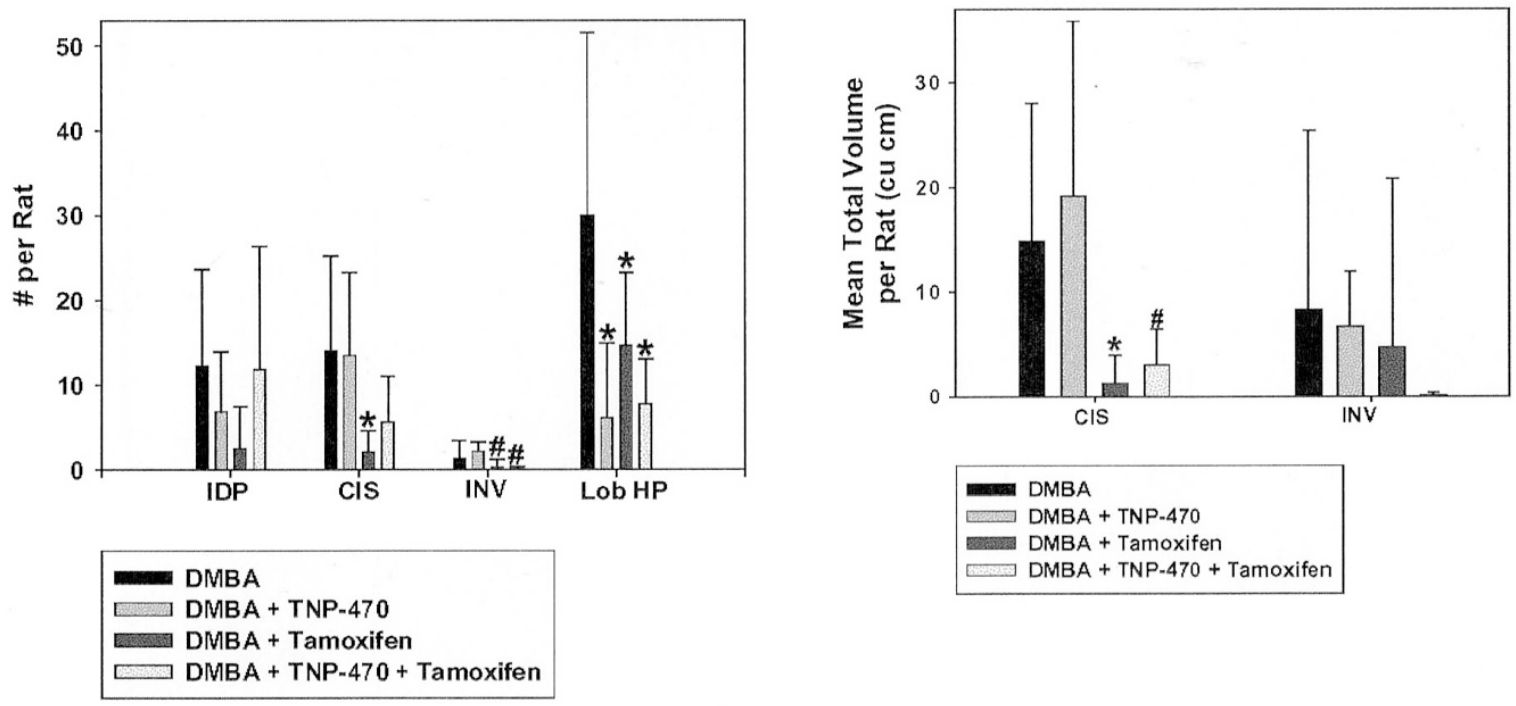

A
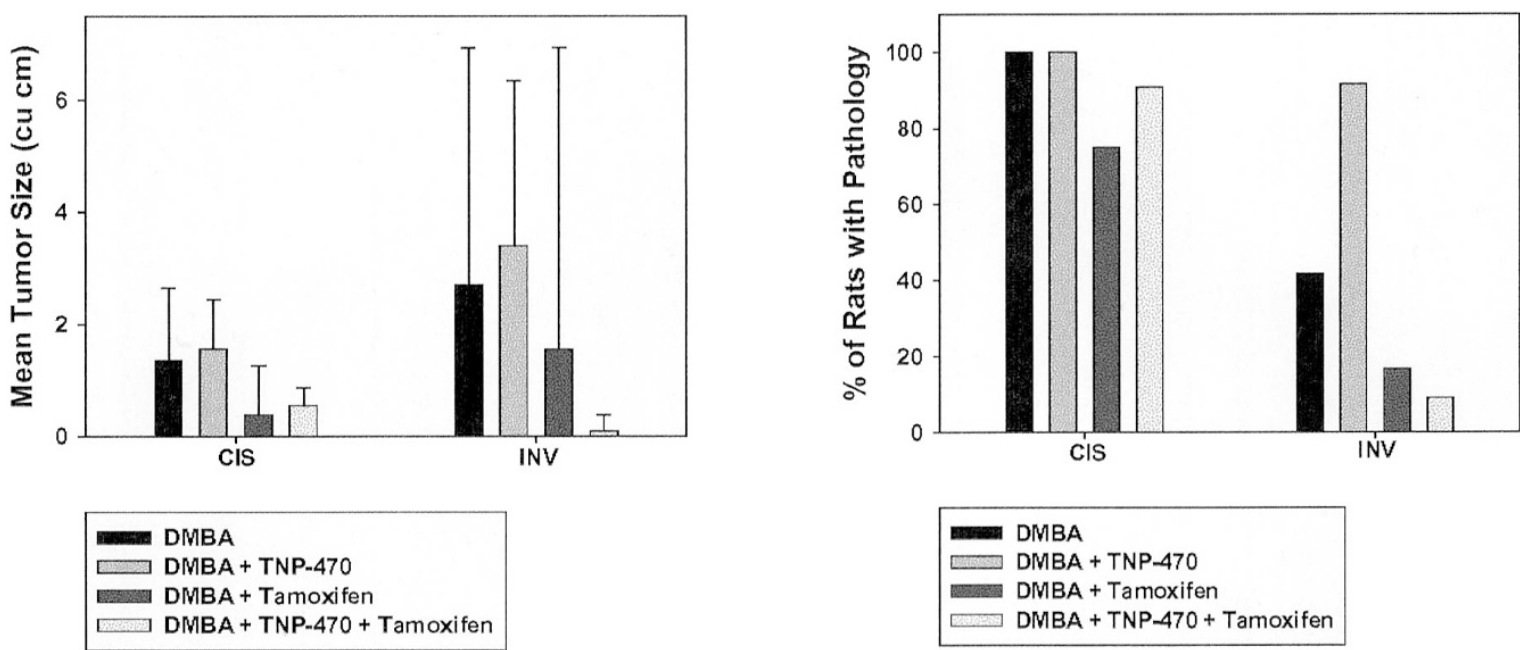

C

Figure 7.

Lack of TNP-470 inhibition of tumor formation with late intervention. Data represent mean number of pathologies ( $\pm \mathrm{SD}$ ) from each treatment group: DMBA alone (DMBA), DMBA and $30 \mathrm{mg} / \mathrm{kg}$ TNP-470 (DMBA + TNP-470), DMBA and $2.5 \mathrm{mg} / \mathrm{kg}$ tamoxifen (DMBA + Tamoxifen), DMBA and $30 \mathrm{mg} / \mathrm{kg}$ TNP-470 and $2.5 \mathrm{mg} / \mathrm{kg}$ tamoxifen $\left(D M B A+T N P-470+\right.$ Tamoxifen). TNP-470 and tamoxifen were delivered 6 weeks after DMBA. administration. ${ }^{*}$ Significantly different from DMBA alone treatment; \#significantly different from TNP-470. Plotted are number of each pathology per rat (A), total tumor volume per rat (B), mean tumor size per rat (C), and the percentage of rats bearing each type of pathology (D).

the sensitivity of vessels in IDP and CIS/INV to endostatin may differ. Differential sensitivity to TNP-470 by individual vascular beds in our studies could play a role in the efficacy of TNP-470 and at least partially account for the lack of tumor inhibition seen when TNP-470 is delivered late in the process of tumorigenesis.

The inability of TNP-470 to block histologic progression to INV is consistent with data from the Rip-1-Tag islet carcinogenesis system (Hanahan, 1985), in which a subset of hyperplastic islets stochastically "switch on" angiogenesis followed by progression to CIS and INV. Using this system, Bergers et al (1999) showed that there was a precursor stage-dependent efficacy of four different angiogenic inhibitors, none of which were able to prevent histologic progression to invasive disease.

\section{Tamoxifen's Mechanisms of Action Are Complex}

Tamoxifen represents a completely different paradigm in this model system, acting directly to inhibit epithelial growth. In the DMBA model system, estrogen is known to stimulate VEGF production (Nakamura et al, 1996). Although tamoxifen stimulates VEGF production in breast cancer cell lines in vitro (Ruohola et al, 1999), the effect of long-term tamoxifen treatment on VEGF is unknown. A recent study in which tamoxifen 


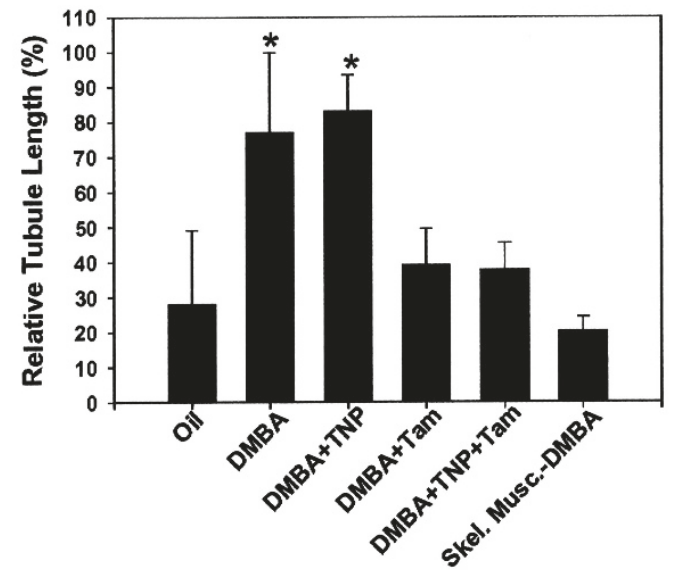

$\mathbf{A}$

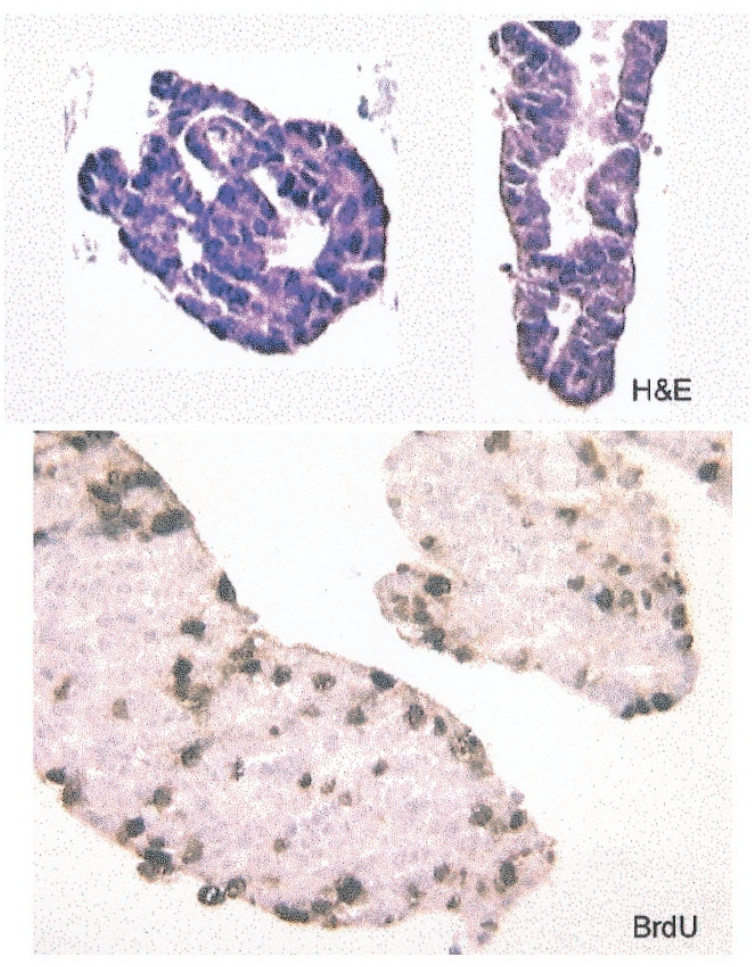

Figure 8.

A, In vitro tubulogenesis assay with isolated normal mammary organoids or skeletal muscle. Twelve weeks after DMBA exposure, normal mammary organoids were isolated from half of the mammary glands from each rat and their angiogenic potential was evaluated as the induction of endothelial tubule formation in a three-dimensional collagen gel assay system. Data are plotted as endothelial tubule length relative to tubule length induced by vascular endothelial cell growth factor (VEGF) set as $100 \%$. Data represents the mean $( \pm \mathrm{SD})$ of five wells of mammary organoids isolated from four rats per group treated with either corn oil (Oi), DMBA alone (DMBA), DMBA and $30 \mathrm{mg} / \mathrm{kg}$ TNP-470 (DMBA+TNP), DMBA and $2.5 \mathrm{mg} / \mathrm{kg}$ tamoxifen (DMBA+Tam), or DMBA and $30 \mathrm{mg} / \mathrm{kg}$ TNP-470 and $2.5 \mathrm{mg} / \mathrm{kg}$ tamoxifen (DMBA+TNP$470+T a m)$. All treatments began within 1 week of DMBA administration. Skeletal muscle from DMBA-treated rats (Skel. Musc.-DMBA) served as negative control. *Significant difference $(p<0.001)$ from control rats (oil) or skeletal muscle. Both tamoxifen and TNP-470 plus tamoxifen in vivo treatments inhibited the angiogenic potential induced by DMBA initiation. B, Histologic analysis of a typical rat mammary organoid preparation. Shown are organoids stained with hematoxylin and eosin (top) or for BrdU (bottom). Few stromal cells can be identified. The distribution of BrdU indicates viability throughout each organoid. Digital images were taken using a $\times 40$ objective. was used as neoadjuvant therapy highlights the complexity of the role of tamoxifen in tumor vascularization (Marson et al, 2001), in that microvascular counts actually increased in $22 \%$ of responding and $57 \%$ of nonresponding tumors. Therefore, predictions regarding tamoxifen's effect on tumor vasculature are tenuous, at best, and microvascular density has been found to be a poor predictor of antiangiogenic efficacy (Folkman, 2001). We chose a dose of tamoxifen that had limited direct effects on the endothelium. Tamoxifen robustly inhibited the formation of CIS and INV in this model system, whether delivered early or late in tumor development. However, the combination of tamoxifen and TNP-470 did not lead to a reduction greater than either alone. We do not believe TNP-470 affected tamoxifen's activity level in our studies for the following reasons. In mice, coadministration of TNP470 and tamoxifen showed no differences in tamoxifen concentrations or that of its metabolites relative to tamoxifen alone (Takei et al, 2000). Furthermore, our organoid data from the TNP-470 + tamoxifen cohort showed that TNP-470 did not abrogate the efficacy of tamoxifen inhibition of angiogenic potential. One obvious hypothesis to explain the lack of an additive effect on tumorigenesis is that tamoxifen delivery to the tumor is inhibited by chronic angiogenic inhibition; however, we have no direct data supporting this conjecture. Whether other biologic interactions affecting the activity of one in the presence of the other were present is unknown.

\section{TNP-470-Resistant Tumors Have a Decreased Apoptotic Rate}

Gross examination of the tumors in each group during treatment made it clear that the growth rate of tumors in the TNP-470 and combined treatment groups were comparable in size to those of the DMBA controls. Therefore, the rare tumors that are "resistant" to inhibition of angiogenesis grew as if no inhibitor were present. Microvascular density, vascular proliferation, and epithelial proliferation did not vary among these groups. However, TNP-470 led to a decreased epithelial apoptotic rate, a finding seen by others (Sano et al, 2002). Mechanisms for this could include a direct effect of TNP-470 on the epithelium, an epithelial response to chronic vascular dysfunction, or a selection of epithelial cells with a decreased apoptotic rate with tumor growth. Whether any of these are operative in the current studies is not yet clear.

\section{Inhibition of Angiogenesis Can Prevent Hormone-Dependent Mammary Tumors}

Currently the class of selective estrogen receptor modulators, such as tamoxifen, is well documented in the prevention of breast tumor formation, recurrence, and metastasis in women (Wickerham, 2002). However, because of the pleiotropic effects of estrogens on cardiovascular, bone, and neural function, alternative and combinatorial methods of chemoprevention will be necessary for some women. The current stud- 
ies provide proof-of-principle that inhibition of angiogenesis could prevent mammary tumor formation in a hormone-sensitive model system. However, the stage of TNP-470 sensitivity is early in tumorigenesis. Under the current conditions, synergism with tamoxifen was not apparent. We identified one other report in which angiogenic inhibition by an anti-inflammatory compound, linomide, decreased carcinogen-induced mammary carcinoma formation (Joseph et al, 1996). No data were reported regarding the role of linomide on hyperplastic or CIS pathologies. Although the mechanism of angiogenic inhibition in this study is indirect, these data support the concept that inhibition of angiogenesis may be a viable method for breast cancer chemoprevention.

\section{Summary}

To our knowledge these are the first studies showing that inhibition of angiogenesis, as a sole mechanism, can prevent the formation of breast cancer. These studies give proof-in-principle that formation of precursor pathologies are dependent upon angiogenesis. Additional studies are required to determine whether all angiogenic inhibitors function at the same stage of progression.

\section{Methods}

\section{Matrigel Pellet Assay}

Virgin female Sprague-Dawley rats (Harlan, Indianapolis, Indiana), 50 to 55 days old, were anesthetized briefly with inhaled isoflurane during the sc injection of $0.5 \mathrm{ml}$ of Matrigel (Becton Dickinson, Bedford, Massachusetts) and $12.8 \mathrm{U} / \mathrm{ml}$ heparin (Elkins-Sinn, Cherry Hill, New Jersey) (Vukanovic et al, 1993). Rats were treated for 14 days with $30 \mathrm{mg} / \mathrm{kg}$ TNP-470, either sc or ip, three times per week; with $15 \mathrm{mg} / \mathrm{kg}$ TNP-470 sc five times per week; with $2.5 \mathrm{mg} / \mathrm{kg}$ tamoxifen in an oil droplet once per week (low dose); or with $1 \mathrm{mg}$ of tamoxifen per rat (high dose) once per week, and the pellets processed for histologic examination. Sections $(6 \mu \mathrm{m})$ were stained for vessels by an antibody to transglutaminase (Thomazy and Fesus, 1989). Three $\times 20$ fields per pellet were analyzed morphometrically for vascular content from two Matrigel pellets per rat. Group identity was blinded for analysis. Mean vascularity per group was compared by one-way ANOVA.

\section{DMBA Tumorigenesis}

Virgin female Sprague-Dawley rats (Taconic Farms, Germantown, New York), 45 days old, were given 8 $\mathrm{mg}$ of DMBA/100 $\mathrm{g}$ body weight $(16 \mathrm{mg} / \mathrm{ml}$ in corn oil at $95-96^{\circ} \mathrm{C}$ covered) or an equal volume of corn oil as a single gavage. TNP-470 and/or tamoxifen treatments began within the first week or 6 weeks after DMBA and continued throughout the duration of each study. Animals were palpated twice weekly for tumors. For purposes of this discussion, the term "tumor" is defined as CIS or INV regardless of size. Tumors may then be either palpable or microscopic. BrdU (50 $\mathrm{mg} / \mathrm{kg}$ ) was administered ip to all animals at 24 and 12 hours prior to death (12 weeks after DMBA). Mammary tissue from all six glands on one side were dissected and pooled for analysis, one side for histologic examination and one side for organoid isolation. Gross tumors and hyperplastic patches were processed separately. Grossly normal mammary glands were dissected free of the pelt. The three abdominal/inguinal glands and the three cervical/thoracic glands were divided into five cassettes, spread thinly over sponges, and processed flat. Previous studies doing extensive levels throughout the block showed that a single histologic section near the center of the 1-mmthick glandular section rarely missed the presence of a patch of proliferative lesions and never missed a CIS (not shown). Pathologic diagnoses were according to Russo and Russo (2000) and read blinded to treatment group. Briefly, proliferative lesions were defined as regions of increased cellularity with atypia. Although occasionally found as single atypical alveolar structures, these were most commonly found in clusters indicating a patch of initiation had occurred within a single terminal ductolobular unit. On whole mount these would appear as a small cluster of proliferative "grapes." Just as multiple alveolar structures are counted as one lobule, for this report each cluster of proliferative lesions is counted as one IDP. Cells were of a ductal morphology, in contrast to lobular hyperplasia, which most closely resembled the beginning of a lactating or tubular adenoma. IDP were also characterized by a fibroinflammatory reaction in most cases, a response never seen in lobular hyperplasia. CIS were mostly of the papillary and cribriform type, commonly divided by bridges of connective tissue. These lesions maintained a pushing border, and although occasionally resulted in dermal necrosis, did so via a mechanism of pressure rather than invasion. Cancerization and expansion of adjacent lobules with in situ carcinoma was the norm, forming large expansile tumors absent an invasive component. To be given a diagnosis of INV, there had to be clear evidence of single cells or nests of cells transversing tissues unrelated to the underlying structure of an expanded lobule. All cases of INV were confirmed with cytokeratin immunohistochemistry. As with a diagnosis of sclerosing adenosis or invasive cribriform carcinoma, this distinction was best made at low magnification. All animal procedures were approved by the Institutional Animal Care and Use Committee of the University of Cincinnati.

\section{Immunohistochemistry}

Immunohistochemistry was performed using Ventana 320ES automatic immunostaining, with primary and secondary antibody incubations for 32 minutes at room temperature, as described previously (Heffelfinger et al, 2000). For the primary mAbs, rat adsorbed anti-mouse (Vector, Burlingame, California) was used as the secondary antibody at 1:50. Preimmune rabbit $\lg G$ or isotype-specific mouse $\lg G$ added in place of 
the primary antibody were used as the negative controls. Histologic sections were double stained by immunohistochemistry for von Willebrand factor (VWF) (polyclonal, 1:400; Dako, Carpenteria, California) and BrdU (clone 3D9, 1:500; a gift from Dr. Azra Raza, Rush Presbyterian Hospital, Chicago, Illinois). In addition, cytokeratin (clone C-11, 1:20; Lab Vision, Fremont, California) staining was performed on tumors that were potentially invasive. Matrigel pellets were stained with anti-transglutaminase (1:50, Lab Vision). Slides for cytokeratin or transglutaminase staining were pretreated in $10 \mathrm{~mm}$ borate at $75^{\circ} \mathrm{C}$ overnight before the addition of the primary antibody.

\section{Terminal Deoxynucleotidyl Transferase-Mediated dUTP Nick-End Labeling (TUNEL) Labeling}

Deparaffinized slides were labeled to identify apoptotic cells using the TUNEL labeling kit from Boehringer Mannheim (Indianapolis, Indiana) according to the manufacturer's instructions and counterstained in Mayer's hematoxylin.

\section{Morphometric Analysis of Tissue Pathology}

Determination of microvascular density, BrdU-positive vascularity, and epithelial proliferation were as described previously (Heffelfinger et al, 2000). Briefly, two digital images ( $\times 10$ objective) in the most vascular region of each pathology were assessed per lesion. The area of vessels was quantified relative to the total area of the lesion in the field using Image Pro Plus (Media Cybernetics, Silver Springs, Maryland). Microvascular density was defined as the number of VWFpositive objects (brown) divided by the epithelial area (EA) per field. Vascular area was determined by the area of VWF staining in vessels without or with BrdU (blue) overlap. The sum of these two areas is total vascular area. All vascular areas were normalized to the EA within the field. Epithelial proliferation was defined by the BrdU-positive area within the EA divided by the EA. In all cases means for each group were compared by one-way ANOVA (multiple group comparison).

Analysis of percent apoptotic cells was determined for each slide from the three most apoptotic $\times 20$ objective fields from 10 tumors per treatment group. Color channel extraction to identify the hematoxylin counterstain was used along with multiple passes with dilation and erosion filters to identify total nuclear count within a predefined mask of the epithelial area. Apoptotic cells were identified by threshold analysis.

\section{Organoid Isolation}

Mammary glands were finely minced using scalpels and digested overnight at $37^{\circ} \mathrm{C}$ in $100 \mathrm{U} / \mathrm{ml}$ collagenase (Life Technologies, Gaithersburg, Maryland) and $150 \mathrm{U} / \mathrm{ml}$ hyaluronidase (Sigma, St. Louis, Missouri) in DMEM/F12 (Mediatech, Herndon, Virginia). The next day epithelial organoids, digested free of adjacent connective tissue, were centrifuged at $2000 \times g$ for 10 minutes. Organoids were washed free of contaminat- ing skeletal muscle and vascular fragments by repeated suspension and resedimentation in DMEM/ F12. Purity of organoids were ascertained by microscopy.

\section{In Vitro Tubulogenesis Assay}

Freshly isolated organoids, tumor pieces, or skeletal muscle pieces of organoid size were placed on the surface layer of a collagen type I sandwich, $2 \mathrm{mg} / \mathrm{ml}$ (Becton Dickinson), in which $4 \times 10^{4}$ HUVEC (Clonetics, San Diego, California) per well had been cultured in a 48-well dish. Organoids in each well are handpicked from a dish under $\times 10$ visualization to assure an equal distribution and to avoid stromal contamination and the presence of any hyperplastic epithelium. Fifteen hours later 3-[4,5-dimethylthiazole-2-yl]-2,5diphenyltetrazolium bromide (MTT) (1 mg/ml) (Sigma) was added. Two hours later the wells were rinsed and fixed in 10\% formalin. Organoids were removed and checked for viability and equality of distribution among replicate wells by MTT staining. Digital images of five replicate wells were analyzed for total area and length of tubules and normalized against medium alone and medium with $100 \mathrm{ng} / \mathrm{ml}$ VEGF (Becton Dickinson).

\section{Statistical Methods}

All statistical evaluations were performed using SigmaStat (SPSS, Chicago, Illinois). Group mean comparisons were by one-way ANOVA, two sided, using 95\% confidence intervals. Two group comparisons were by Student's $t$ test.

\section{Acknowledgements}

The authors thank Ms. Lynn McKee and Ms. Janice Armstrong for their excellent technical support in immunohistochemistry. In addition, we thank Dr. Jose Russo for his time at the microscope helping us to learn his nomenclature, Dr. David Askew for his tireless review of this manuscript, and TAP Holdings, Inc. for the TNP-470.

\section{References}

Bergers G, Javaherian K, Lo K-M, Folkman J, and Hanahan D (1999). Effects of angiogenesis inhibitors on multistage carcinogenesis in mice. Science 284:808-812.

Folkman $J$ (1990). What is the evidence that tumors are angiogenesis dependent? J Natl Cancer Inst 82:4-6.

Folkman J (2001). Angiogenesis-dependent diseases. Semin Oncol 28:536-542.

Folkman J, Watson K, Ingber D, and Hanahan D (1989). Induction of angiogenesis during the transition from hyperplasia to neoplasia. Nature 339:58-61.

Gagliardi A, Hennig B, and Collins D (1996). Antiestrogens inhibit endothelial cell growth stimulated by angiogenic growth factors. Anticancer Res 16:1101-1106. 
Hanahan D (1985). Heritable formation of pancreatic beta-cell tumours in transgenic mice expressing recombinant insulin/ simian virus 40 oncogenes. Nature 315:115-122.

Heffelfinger S, Yassin R, Miller M, and Lower E (1996). Vascularity of proliferative breast disease and carcinoma in situ correlates with histologic features. Clin Cancer Res 2:1873-1878.

Heffelfinger SC, Gear RB, Taylor K, Schneider J, LaDow K, Miller MA, and Warshawsky D (2000). DMBA-induced preinvasive mammary tumors are angiogenic in vitro and in vivo. Lab Invest 80:485-492.

Hollingsworth A, Lerner M, Lightfoot S, Wilkerson K, Hanas J, McCay P, and Brackett D (1998). Prevention of DMBAinduced rat mammary carcinomas comparing leuprolide, oophorectomy, and tamoxifen. Breast Cancer Res Treat 47:63-70.

Huggins C, Grand L, and Brillantes F (1961). Mammary cancer induced by a single feeding of polynuclear hydrocarbons, and its suppression. Nature 189:204-207.

Ingber D, Fujita T, Kishimoto S, Sudo K, Kanamaru T, Brem $\mathrm{H}$, and Folkman J (1990). Synthetic analogues of fumagillin that inhibit angiogenesis and suppress tumour growth. Nature 348:555-557.

Jordan V and Allen K (1980). Evaluation of the antitumour activity of the non-steroidal antioestrogen monohydroxytamoxifen in the DMBA-induced rat mammary carcinoma model. Eur J Cancer 16:239-251.

Joseph I, Vukanovic J, and Isaacs J (1996). Antiangiogenic treatment with linomide as chemoprevention for prostate, seminal vesicle, and breast carcinogenesis in rodents. Cancer Res 56:3404-3408.

Kerbel R, Viloria-Petit A, Okada F, and Rak J (1998). Establishing a link between oncogenes and tumor angiogenesis. Mol Med 4:286-295.

Kohn E and Liotta L (1995). Molecular insights into cancer invasion: Strategies for prevention and intervention. Cancer Res 55:1856-1862.

Kruger E and Figg W (2000). TNP-470: An angiogenesis inhibitor in clinical development for cancer. Expert Opin Investig Drugs 9:1383-1396.

Lichtenbeld $H$, Barendsz-Janson A, van Essen $H$, Struijker Boudier Griffioen A, and HF H (1998). Angiogenic potential of malignant and non-malignant human breast tissues in an in vivo angiogenesis model. Int $\mathrm{J}$ Cancer 77:455-459.

Marson L, Kurian K, Miller W, and Dixon J (2001). The effect of tamoxifen on breast tumour vascularity. Breast Cancer Res Treat 66:9-15.
Nakamura J, Savinov A, Lu Q, and Brodie A (1996). Estrogen regulates vascular endothelial growth/permeability factor expression in 7,12 dimethylbenz(a)anthracene-induced rat mammary tumors. Endocrinology 137:5589-5596.

Perletti G, Concari P, Giardini R, Marras E, Piccinini F, Folkman J, and Chen L (2001). Antitumor activity of endostatin against carcinogen-induced rat primary mammary tumors. Cancer Res 60:1793-1796.

Ruohola JK, Valve EM, Karkkainen MJ, Joukov V, Alitalo K, and Harkonen PL (1999). Vascular endothelial growth factors are differentially regulated by steroid hormones and antiestrogens in breast cancer cells. Mol Cell Endocrinol 149:2940.

Russo J and Russo I (2000). Atlas and histologic classification of tumors of the rat mammary gland. J Mammary Gland Biol Neoplasia 5:187-200.

Russo J, Russo I, van Zwieten M, Rogers A, and Gusterson B (1989). Classification of neoplastic and nonneoplastic lesions of the rat mammary gland. In: Jones TC, Mohr U, and Hunt RD, editors. Integument and mammary glands. Berlin, New York: Springer-Verlag, 275-304.

Sano B, Sugiyama K, Sano J, and Saji S (2002). Antitumor effects induced by the combination of TNP-470 as an angiogenesis inhibitor and Lentinan as a biological response modifier in a rabbit spontaneous liver metastasis model. Surg Today 32:503-509.

Takechi A, Uozumi T, Kawamoto K, Ito A, Kurisu K, and Sudo K (1994). Inhibitory effect of TNP-470, a new anti-angiogenic agent, on the estrogen induced rat pituitary tumors. Anticancer Res 14:157-162.

Takei H, Lee E, Cisneros A, and Jordan V (2000). Effects of angiogenesis inhibitor TNP-470 on tamoxifen-stimulated MCF-7 breast tumors in nude mice. Cancer Lett 155:129135.

Thomazy V and Fesus L (1989). Differential expression of tissue transglutaminase in human cells: An immunohistochemical study. Cell Tissue Res 255:215-224.

Vukanovic J, Passaniti A, Hirata T, Traystman R, Hartley-Asp $B$, and Isaacs J (1993). Antiangiogenic effects of the quinoline-3-carboxamide linomide. Cancer Res 53:18331837.

Wickerham L (2002). Tamoxifen: An update on current data and where it can now be used. Breast Cancer Res Treat 75(Suppl 1):S7-S12. 